\title{
Southern IRDCs seen with Spitzer/MIPS
}

\author{
H. Linz ${ }^{1}$, Ra. Klein ${ }^{2}$, L. Looney ${ }^{3}$, Th. Henning ${ }^{1}$, B. Stecklum ${ }^{4}$ \\ and L. $-\AA$. Nyman ${ }^{5}$ \\ ${ }^{1}$ MPIA Heidelberg, Germany ${ }^{2}$ MPE Garching, Germany \& UC Berkeley, USA \\ ${ }^{3}$ University of Illinois, Urbana-Champagne, USA ${ }^{4}$ TLS Tautenburg, Germany ${ }^{5}$ ESO, Chile
}

\begin{abstract}
Infrared dark clouds (IRDCs) are generally assumed to be a promising hunting ground for tracing very early stages of massive star formation. Observations with Spitzer are a viable tool to probe their interiors that are still dominated by strong dust extinction even at $8 \mu \mathrm{m}$. With Spitzer/MIPS, we have observed several IRDCs at 24 and 70 micron. We generally find weak 24 micron sources within the IRDCs. However, at 70 micron these sources remain weak and thus indicate lower luminosities at the current state of evolution. Indications for internal substructures exist, separating regions with compact IR sources from even more dark regions.
\end{abstract}

Keywords. infrared: ISM, stars: formation, ISM: individual (G316.719+0.073)

Based on examining MSX $8.3 \mu \mathrm{m}$ images to identify high-contrast IRDCs in the southern hemisphere, several candidates have been mapped with the SIMBA 1.2-mm bolometer at the SEST in 2001 and 2003. The vast majority of the mapped IRDCs shows clear coincidence of 1.2-mm emission with the often filamentary mid-infrared extinction structures. The IRDC shown in Fig. 1 is still surprisingly dark in the northern tip even at $24 \mu \mathrm{m}$, but is associated with a small cluster of deeply embedded MIR sources, comprising fluxes of $5-75$ mJy. Only one weak source with $\sim 0.7$ Jy remains at $70 \mu \mathrm{m}$. Located at roughly 3 $\mathrm{kpc}$ distance, the IRDC mass is $400-950 \mathrm{M}_{\odot}$ (depending on the extinction law), derived from the MIR extinction. Complementary, the mm emission can be translated to a total mass of $561 \mathrm{M}_{\odot}$ (assuming $\left.T_{\text {Dust }}=20 \mathrm{~K}, \kappa_{1.2 \mathrm{~mm}}=1 \mathrm{~cm}^{2} \mathrm{~g}^{-1}\right)$. In the future, high spatial resolution mm observations have priority, a task for the ATCA 3-mm system.
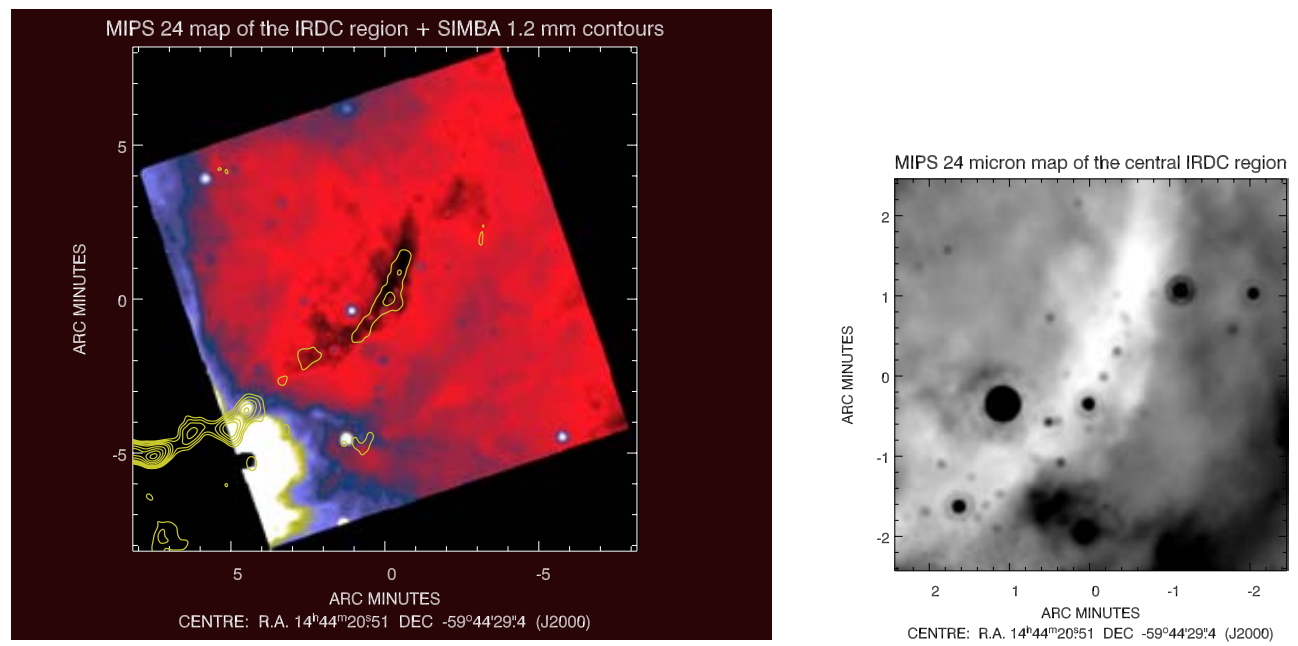

Figure 1. Spitzer/MIPS $24 \mu \mathrm{m}$ view on IRDC G316.719+0.073. Left: With 1.2-mm contours which also trace the more evolved massive star-forming complex to the south-east. Right: Inverse grey-scale map exclusively of the IRDC, emphasising the embedded 24 micron sources. 